\title{
Electromechanical Coupled Non-linear Dynamics for Micro Plate Simply supported on four Sides
}

\author{
Libo Sun \\ College of Environmental and Chemical Engineering, \\ Hebei Key Laboratory of Applied Chemistry \\ Yanshan University \\ Qinhuangdao, China, 066004
}

\author{
Lizhong Xu \\ Mechanical Engineering Institute \\ Yanshan University \\ Qinhuangdao, China, 066004 \\ xlz@ysu.edu.cn
}

\begin{abstract}
Dynamic characteristic of the micro plate under the micro scale effect shows non-linear features, traditional linear analysis methods can not satisfy the design precision of MEMS system, and non-linear dynamics analysis method provides an effective solution. In this paper, based on the small deflection theory and electrostatics theory, take typical boundary condition of the micro plate simply supported on four sides as example, the basic differential equation of lateral vibration is established, using Linz Ted-Poincare method, displacement and time course of forced vibration, time responses of forced vibration are calculated. The research results provide theoretical basis for optimizing the system parameters, improving the ability to resist interferences and increases the reliability.
\end{abstract}

Keywords- micro plate; MEMS; non-linear dynamics; small deflection theory; Linz Ted-Poincare method

\section{INTRODUCTION}

Within the microscopic scale, machinery can be divided into small machinery dimensions of $1 \sim 10 \mathrm{~mm}$, micro machinery dimensions of $1 \mu \mathrm{m} \sim 1 \mathrm{~mm}$, and nano machinery of $1 \mathrm{~nm} \sim 1 \mu \mathrm{m}$ according to the different characteristics scale. Micro-electromechanical systems is called Micro machine and MEMS system, which generally consists of micro machinery and nano machinery, but not the macro machinery be miniaturized simply. Micro-electromechanical system (MEMS) can be described as micron even smaller order microcomponent or micro system made up of electron or mechanical component, and its processing method and driving principle are different from macroscopic machine. Such devices are new technology products which can realize higher accuracy, more convenient and efficient, cheaper and better energy saving micro operation, also, can feel, understand, reform and control objective world. MEMS have potential applications in automatic control, mobile communication, aerospace instrument and biomedical engineering field ${ }^{[1 \sim 3]}$.

Micro components are important parts, which are composed of micro agencies and micro-electromechanical systems. Although, structure of the micro components is generally simple, we can use their displacement, deformation and movement to realize the function of micro sensor and micro actuator. Main form of the micro components are consist of micro plate, micro beam, micro film and micro hinge etc. Among them, as a typical structural element, the

The assistances from the National Natural Science Foundation of China (No. 51275441) and Science Research Plan of Hebei Education Department (No. Z2012031) for this work are heartily acknowledged. micro plate has been widely used in MEMS technology, such as micro valve, micro pump and micro sensor etc. At present, for the dynamic characteristic of the micro plate, especially under the micro scale effect, the research on the vibration of electromechanical coupled micro plate is still very few. However, these researches would have a positive impact on structural design, optimization, control and improving the stability and reliability of MEMS devices and systems.

In the micro plate electromechanical coupled system, we utilize the electrostatic field which has been applied on fixed driving plate and micro mobile plate to generate electrostatic field driving force. The electrostatic field driving force would cause the mutual attraction between the fixed driving plate and the micro mobile plate, the micro mobile plate would displace relatively due to the attraction, under alternating electric field force, and the high frequency vibration of the micro mobile plate would be generated. Displacement of the micro mobile plate would be changed with the changes of the electrostatic field driving force frequencies, when driving frequency closes to natural frequency of the micro mobile plate, the micro mobile plate would resonate, by this time, the displacement maximum of the micro mobile plate is reached.

In this paper, based on the small deflection theory and electrostatics theory, the electromechanical coupled dynamics model is established, formulas of the static and dynamic electric field force are derived, and moreover, the basic differential equation of lateral vibration is established by using elasticity theory. Take typical boundary condition of the micro plate as example, displacement and time course of forced vibration, time responses of forced vibration are calculated with the aid of computing simulation to achieve the systemic analysis and design, which provides theoretical basis for optimizing the system parameters, improving the ability to resist interferences and increases the reliability.

\section{DYNAMIC MODEL OF ELECTROMECHANICAL COUPLED MICRO PLATE}

Fig.1 illustrates a dynamic model of electromechanical coupled micro plate, consisting of mechanical system, electrical system, and coupled part. The mechanical system is a micro plate subjected to electric field force which distributes equally on the micro plate. The upper is the micro mobile plate, it is the main object of study, we call it micro plate, the below 
is the fixed driving plate, the DC electrostatic bias voltage is applied between the two plates. The electrical source, resistance and capacitance construct electrical system. Mechanical system and electrical system achieve electromechanical coupled state by electric field force. The micro plates system obey the basic assumptions of elastic thin plat small deflection theory, as follows

- Middle surface normal hypothesis, that is to say, the middle surface normal maintains a line and always be vertical to the middle surface throughout the process of deformation.

- The normal stress of the middle surface is neglected.

- Without the deformation inside the middle surface.

- Inertial forces should be included in but moment of inertia should be ignored ${ }^{[9]}$.

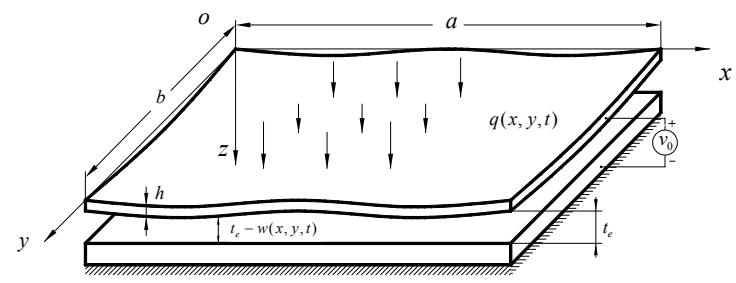

Fig.1 Dynamic model of electromechanical coupled micro plate

The micro plate simply supported on four sides is widely used in micro pump membrane; fig.2 illustrates the Cartesian coordinate system of micro plate simply supported on four sides.

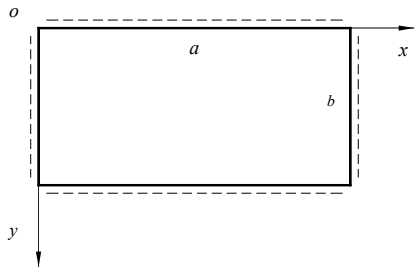

Fig.2 Cartesian coordinate system of micro plate simply supported on four sides

\section{DYNAMIC DIFFERENTIAL EQUATIONS}

Based on the thin plate deflection theory, the control differential equation of the deflection surface subjected to electric field force is given by

$$
\frac{\partial^{4} w}{\partial x^{4}}+\frac{2 \partial^{4} w}{\partial x^{2} \partial y^{2}}+\frac{\partial^{4} w}{\partial y^{4}}+\frac{\rho h}{D} \frac{\partial^{2} w}{\partial t^{2}}=\nabla^{2} \nabla^{2} w+\frac{\rho h}{D} \frac{\partial^{2} w}{\partial t^{2}}=\frac{q}{D}(x, y, t)
$$

Here $\mathrm{w}$ is transverse displacement of the micro plate, $\mathrm{D}$ is the flexural rigidity of the micro plate material, (e.g. $D=E_{t} h^{3} / 12\left(1-v^{2}\right)$, where $E_{\mathrm{t}}$ and $v$ are the elastic modulus and Poisson ratio of the micro plate material respectively, and $h$ is the thickness of the micro plate), $\nabla^{2} \nabla^{2}$ is biharmonic operator, $\nabla^{2} \nabla^{2}=\partial^{4} / \partial x^{4}+2 \partial^{4} / \partial x^{2} \partial y^{2}+\partial^{4} / \partial y^{4}, \rho$ is the mass density of the micro plate, and $q$ is the load per unit area above the micro plate. When $q(x, y, t)$ is equal to 0 , the free vibration differential equation of the micro plate is obtained.
When the sinusoidal alternating voltage is applied on the system, (e.g. $\Delta \mathrm{v}_{i s}=U_{0} \cos \omega_{e} t, U_{0}$ is excitation voltage amplitude, $\omega \mathrm{e}$ is excitation voltage frequency), because of positive and negative values canceling each other,the voltage only has effect on the dynamic responses, but not the static displacement. According to reference [10], the dynamic electrostatic force per unit area caused by the voltage fluctuation can be calculated as follows

$$
\Delta q=U_{0} \varepsilon_{0} A \cos \omega_{e} t /\left(t_{0}-w_{0}+d_{c} / \varepsilon_{r}\right)^{2}
$$

Here $\varepsilon_{0}$ is the permittivity of free space, equal to $\varepsilon_{0}=8.85 \times 10^{-12} \mathrm{C}^{2} \cdot \mathrm{N}^{-1} \cdot \mathrm{m}^{-2}, \quad \varepsilon_{\mathrm{r}}$ is the relative dielectric constant of the upper micro plate coating, $d_{\mathrm{c}}$ is the thickness of the upper micro plate coating, $t_{0}$ is the initial clearance between two micro plates, and $w_{0}$ is the static average displacement along $z$-axis of micro plate.

The basic equation of the forced vibration for micro plate under the harmonic voltage exciting is

$$
D \nabla^{2} \nabla^{2} w+\rho h \partial^{2} w / \partial t^{2}=U_{0} \varepsilon_{0} A \sin \omega_{e} t /\left(t_{0}-w_{0}+d_{c} / \varepsilon_{r}\right)^{2}
$$

\section{NON-LINEAR FORCED VIBRATION}

When apply the periodic exciting voltage between the micro plate and the fixed driving plate, the dynamic electrostatic force per unit area caused by the voltage fluctuation can be calculated by using Eq. (2). Introducing the dimensionless small parameter $\varepsilon$, as the static average displacement $\bar{w}_{0}$ is small, $\bar{w}_{0} /\left(t_{0}+d_{c} / \varepsilon_{r}\right)$ can be taken as small parameter $\varepsilon$, and the parameter is assumed to be small, $\varepsilon=\bar{w}_{0} /\left(t_{0}+d_{c} / \varepsilon_{r}\right),(0<\varepsilon<1)$. Let the exiting force amplitude engendered by exciting voltage and small parameter $\varepsilon$ is the same order of magnitude. Then, Eq. (2) can be defined in Taylor series form (neglect high-order terms) as follows

$$
\begin{gathered}
\Delta q=\frac{U_{0}^{2} \varepsilon_{0} \Delta w}{\left(t_{0}-\bar{w}_{0}+d_{c} / \varepsilon_{r}\right)^{3}}+\frac{3 U_{0}^{2} \varepsilon_{0} \Delta w^{2}}{\left(t_{0}+d_{c} / \varepsilon_{r}\right)^{3} \bar{w}_{0}} \varepsilon+ \\
\frac{6 U_{0}^{2} \varepsilon_{0} \Delta w^{3}}{\left(t_{0}+d_{c} / \varepsilon_{r}\right)^{4} \bar{w}_{0}} \varepsilon+\frac{U_{0} \varepsilon_{0} E_{0} \cos \omega_{e} t}{\left(t_{0}-\bar{w}_{0}+d_{c} / \varepsilon_{r}\right)^{2}}
\end{gathered}
$$

Substituting equation (4) into equation (1), neglect highorder terms, and let $\Delta w(x, y, t)=\phi(x, y) q(t)$, the non-linear dynamical equation can be obtained

$$
\ddot{q}(t)+q(t)\left[\omega_{0}^{2}-\frac{b_{1}}{\rho h} \varepsilon q(t)-\frac{b_{2}}{\rho h} \varepsilon q^{2}(t)\right]=F_{0} \cos \omega_{e} t
$$

Where $b_{1}=3 U_{0}^{2} \varepsilon_{0} \bar{\phi} /\left[\left(t_{0}+d_{c} / \varepsilon_{r}\right)^{3} \bar{w}_{0}\right]$,

$b_{2}=6 U_{0}^{2} \varepsilon_{0} \bar{\phi}^{2} /\left[\left(t_{0}+d_{c} / \varepsilon_{r}\right)^{4} \bar{w}_{0}\right]$,

$b_{3}=U_{0} \varepsilon_{0} /\left[\left(t_{0}-\bar{w}_{0}+d_{c} / \varepsilon_{r}\right)^{2} \bar{\phi}\right], F_{0}=b_{3} E_{0} /(\rho h)$,

$\bar{\phi} \approx \frac{1}{a b} \int_{0}^{a} \int_{0}^{b} \sum \phi_{i}(x, y) d x d y, \bar{\phi}^{2} \approx \frac{1}{a b} \int_{0}^{a} \int_{0}^{b} \sum \phi_{i}^{2}(x, y) d x d y$.

When $\varepsilon=0$, the Eq. (5) degrades to the linear dynamical equation of derivation system. 


$$
\ddot{q}(t)+\omega_{0}^{2} q(t)=F_{0} \cos \omega_{e} t
$$

According to reference [11], using the Linz Ted-Poincare method, the solution of the equation can be given as follows

$$
q(t, \varepsilon)=q_{0}(t)+\varepsilon q_{1}(t)+\varepsilon^{2} q_{2}(t)+\ldots \ldots
$$

The initial conditions are

$$
\left\{\begin{array}{l}
q_{0}(0)=Q_{0 i}, \dot{q}_{0}(0)=0 \\
q_{1}(0)=0, \dot{q}_{1}(0)=0 \\
q_{2}(0)=0, \dot{q}_{2}(0)=0 \\
\cdots \cdots
\end{array}\right.
$$

Substituting Esq. (7) and (8) into Eq.(5), and then setting the coefficients with same power of $\varepsilon$ to zero, we obtain the linear equations, then using the initial condition (8) to calculate repeatedly, we get the time solution which has enough precision as follows

$$
q(t, \varepsilon)=B_{0} \varepsilon+C_{0} \varepsilon^{2}+\left(Q_{0}+B_{1} \varepsilon+C_{1} \varepsilon^{2}\right) \cos \omega_{e} t+\left(B_{2} \varepsilon+C_{2} \varepsilon^{2}\right) \cos 2 \omega_{e} t+
$$$$
\left(B_{3} \varepsilon+C_{3} \varepsilon^{2}\right) \cos 3 \omega_{e} t+C_{4} \varepsilon^{2} \cos 4 \omega_{e} t+C_{5} \varepsilon^{2} \cos 5 \omega_{e} t
$$

$$
\begin{aligned}
& \text { Where } \begin{array}{c}
B_{0}=b_{1} Q_{0}^{2} / 2 \rho h \omega_{0}^{2} \\
B_{1}=3 b_{2} Q_{0}^{3} \omega_{0}^{2}\left(4 \omega_{e}^{2}-\omega_{0}^{2}\right)\left(9 \omega_{e}^{2}-\omega_{0}^{2}\right) / 4 \rho h M \\
B_{2}=b_{1} Q_{0}^{2} \omega_{0}^{2}\left(\omega_{e}^{2}-\omega_{0}^{2}\right)\left(9 \omega_{e}^{2}-\omega_{0}^{2}\right) / 2 \rho h M \\
B_{3}=b_{2} Q_{0}^{3} \omega_{0}^{2}\left(\omega_{e}^{2}-\omega_{0}^{2}\right)\left(4 \omega_{e}^{2}-\omega_{0}^{2}\right) / 4 \rho h M \\
M=\omega_{0}^{8}-14 \omega_{0}^{6} \omega_{e}^{2}+49 \omega_{0}^{4} \omega_{e}^{4}-36 \omega_{0}^{2} \omega_{e}^{6}
\end{array} \\
& C_{0}=\left[\begin{array}{c}
Q_{0} \omega_{e}^{10}\left(-57600 B_{1} b_{1}-43200 Q_{0} B_{2} b_{2}-86400 Q_{0} B_{0} b_{2}\right)+ \\
Q_{0} \omega_{e}^{6} \omega_{0}^{4}\left(-30580 B_{1} b_{1}-22935 Q_{0} B_{2} b_{2}-45870 Q_{0} B_{0} b_{2}\right)+ \\
Q_{0} \omega_{e}^{4} \omega_{0}^{6}\left(4092 B_{1} b_{1}+3069 Q_{0} B_{2} b_{2}+6138 Q_{0} B_{0} b_{2}\right)+ \\
Q_{0} \omega_{e}^{2} \omega_{0}^{8}\left(-220 B_{1} b_{1}-165 Q_{0} B_{2} b_{2}-330 Q_{0} B_{0} b_{2}\right)+ \\
\left.Q_{0} \omega_{0}^{10}\left(4 B_{1} b_{1}+3 Q_{0} B_{2} b_{2}+6 Q_{0} B_{0} b_{2}\right)\right] / \rho h N
\end{array}\right.
\end{aligned}
$$

$C_{1}=\left[Q_{0} \omega_{e}^{8} \omega_{0}^{2}\left(129600 Q_{0} B_{1} b_{2}+43200 Q_{0} B_{3} b_{2}+115200 B_{0} b_{1}+57600 B_{2} b_{1}\right)+\right.$ $Q_{0} \omega_{e}^{6} \omega_{0}^{4}\left(-60084 Q_{0} B_{1} b_{2}-20028 Q_{0} B_{3} b_{2}-53408 B_{0} b_{1}-26704 B_{2} b_{1}\right)+$ $Q_{0} \omega_{e}^{4} \omega_{0}^{6}\left(8721 Q_{0} B_{1} b_{2}+2907 Q_{0} B_{3} b_{2}+7752 B_{0} b_{1}+3876 B_{2} b_{1}\right)+$

$Q_{0} \omega_{e}^{2} \omega_{0}^{8}\left(-486 Q_{0} B_{1} b_{2}-162 Q_{0} B_{3} b_{2}-432 B_{0} b_{1}-216 B_{2} b_{1}\right)+$

$\left.Q_{0} \omega_{0}^{10}\left(9 Q_{0} B_{1} b_{2}+3 Q_{0} B_{3} b_{2}+8 B_{0} b_{1}+4 B_{2} b_{1}\right)\right] / \rho h N$

$C_{2}=\left[Q_{0} \omega_{e}^{8} \omega_{0}^{2}\left(21600 Q_{0} B_{2} b_{2}+21600 Q_{0} B_{0} b_{2}+14400 B_{1} b_{1}+14400 B_{3} b_{1}\right)+\right.$

$Q_{0} \omega_{e}^{6} \omega_{0}^{4}\left(-26214 Q_{0} B_{2} b_{2}-26214 Q_{0} B_{0} b_{2}-17476 B_{1} b_{1}-17476 B_{3} b_{1}\right)+$

$Q_{0} \omega_{e}^{4} \omega_{0}^{6}\left(4914 Q_{0} B_{2} b_{2}+4914 Q_{0} B_{0} b_{2}+3276 B_{1} b_{1}+3276 B_{3} b_{1}\right)+$

$Q_{0} \omega_{e}^{2} \omega_{0}^{8}\left(-306 Q_{0} B_{2} b_{2}-306 Q_{0} B_{0} b_{2}-204 B_{1} b_{1}-204 B_{3} b_{1}\right)+$

$\left.Q_{0} \omega_{0}^{10}\left(6 Q_{0} B_{2} b_{2}+6 Q_{0} B_{0} b_{2}+4 B_{1} b_{1}+4 B_{3} b_{1}\right)\right] / \rho h N$

$C_{3}=\left[Q_{0} \omega_{e}^{8} \omega_{0}^{2}\left(4800 Q_{0} B_{1} b_{2}+9600 Q_{0} B_{3} b_{2}+6400 B_{2} b_{1}\right)+\right.$

$Q_{0} \omega_{e}^{6} \omega_{0}^{4}\left(-6492 Q_{0} B_{1} b_{2}-12984 Q_{0} B_{3} b_{2}-8656 B_{2} b_{1}\right)+$

$Q_{0} \omega_{e}^{4} \omega_{0}^{6}\left(1827 Q_{0} B_{1} b_{2}+3654 Q_{0} B_{3} b_{2}+2436 B_{2} b_{1}\right)+$
$Q_{0} \omega_{e}^{2} \omega_{0}^{8}\left(-138 Q_{0} B_{1} b_{2}-276 Q_{0} B_{3} b_{2}-184 B_{2} b_{1}\right)+$

$\left.Q_{0} \omega_{0}^{10}\left(3 Q_{0} B_{1} b_{2}+6 Q_{0} B_{3} b_{2}+4 B_{2} b_{1}\right)\right] / \rho h N$

$C_{4}=\left[Q_{0} \omega_{e}^{8} \omega_{0}^{2}\left(2700 Q_{0} B_{2} b_{2}+3600 B_{3} b_{1}\right)+Q_{0} \omega_{e}^{6} \omega_{0}^{4}\left(-3783 Q_{0} B_{2} b_{2}-5044 B_{3} b_{1}\right)+\right.$

$Q_{0} \omega_{e}^{4} \omega_{0}^{6}\left(1197 Q_{0} B_{2} b_{2}+1596 B_{3} b_{1}\right)+Q_{0} \omega_{e}^{2} \omega_{0}^{8}\left(-117 Q_{0} B_{2} b_{2}-156 B_{3} b_{1}\right)+$

$\left.Q_{0} \omega_{0}^{10}\left(3 Q_{0} B_{2} b_{2}+4 B_{3} b_{1}\right)\right] / \rho h N$

$C_{5}=Q_{0}^{2} B_{3} b_{2}\left(1728 \omega_{e}^{8} \omega_{0}^{2}-2460 \omega_{e}^{6} \omega_{0}^{4}+819 \omega_{e}^{4} \omega_{0}^{6}-90 \omega_{e}^{2} \omega_{0}^{8}+3 \omega_{0}^{10}\right) / \rho h N$

$N=4 \omega_{0}^{12}-220 \omega_{0}^{10} \omega_{e}^{2}+4092 \omega_{0}^{8} \omega_{e}^{4}-30580 \omega_{0}^{6} \omega_{e}^{6}+84304 \omega_{0}^{4} \omega_{e}^{8}-57600 \omega_{0}^{2} \omega_{e}^{10}$

Where $\omega_{0}$ is the natural frequency of the system, $\omega_{\mathrm{e}}$ is

the exciting frequency.

\section{ANALYSIS AND DISCUSSIONS OF TYPICAL EXAMPLE}

Take typical boundary condition of the micro plate simply supported on four sides as example, the parameters for the electromechanical coupled system are shown in Table I. Fig.3 shows the time responses of forced vibration. Fig. 4 shows the displacement and time course of forced vibration.

\begin{tabular}{|c|c|c|c|c|}
\hline TABLE \\
\hline The system parameters & $a / \mathrm{mm}$ & $b / \mathrm{mm}$ & $h / \mu \mathrm{m}$ & $t_{0} / \mu \mathrm{m}$ \\
\hline Values & 2 & 1 & 5 & 2 \\
\hline The system parameters & $d_{\mathrm{c}} / \mu \mathrm{m}$ & $\varepsilon_{\mathrm{r}}$ & $E / \mathrm{GPa}$ & $\rho / \mathrm{kg} \cdot \mathrm{m}^{-3}$ \\
\hline Values & 0.5 & 8.4 & 68 & $2.7 \times 10^{3}$ \\
\hline
\end{tabular}

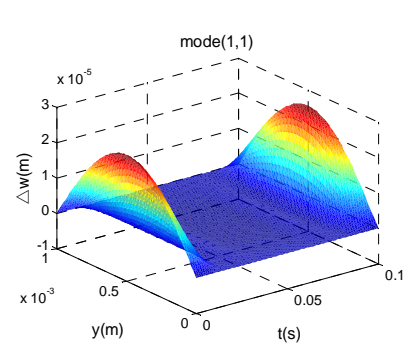

(a) Sectional drawing of $y$-axis

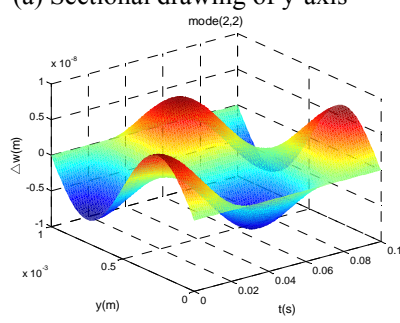

(c) Sectional drawing of y-axis

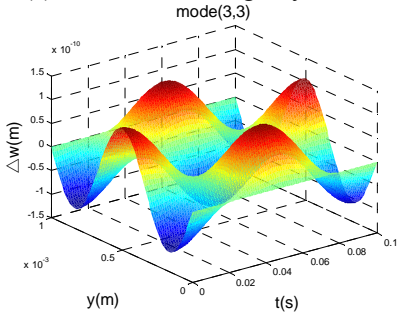

(e) Sectional drawing of y-axis

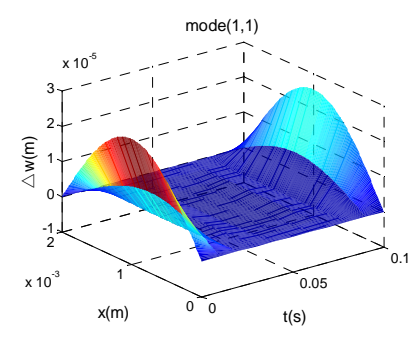

(b) Sectional drawing of $\mathrm{x}$-axis

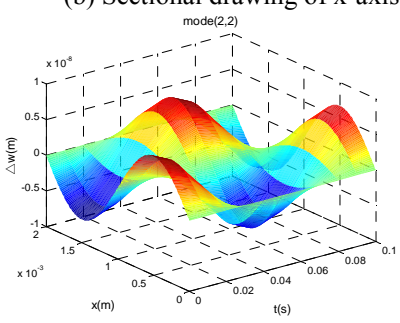

(d) Sectional drawing of $\mathrm{x}$-axis

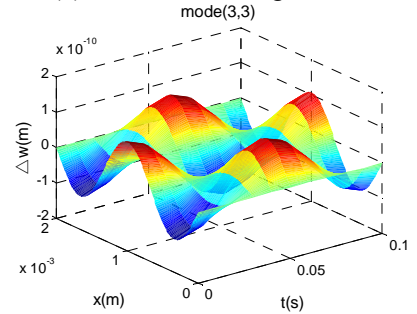

(f) Sectional drawing of $x$-axis
Fig.3 Time responses of forced vibration 


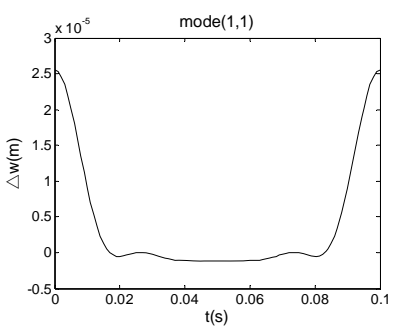

(a) Mode $(1,1)$

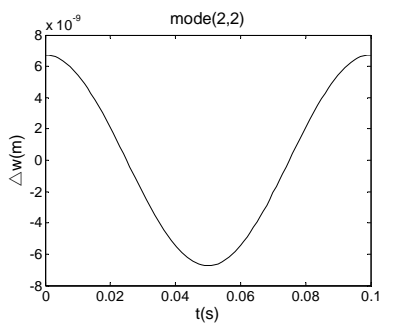

(b) Mode $(2,2)$

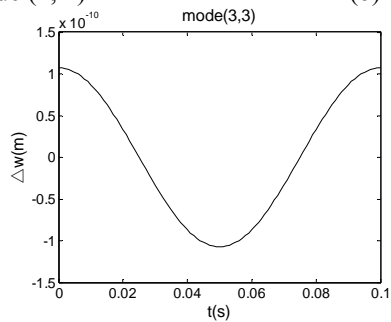

(c) Mode $(3,3)$

Fig.4 Displacement and time course of forced vibration

Eq. (9) is utilized for the analysis of the non-linear forced response of the electromechanical coupled micro plate. The forced response corresponding to the first three modes are given in Fig. 3 and Fig.4, the working voltage between two micro plates $U_{0}=7.0 \mathrm{v}$, the exciting frequency $\omega_{e}=20 \pi \mathrm{rad} / \mathrm{s}$. The maximum dynamic displacement occurs at point ( $x=1 \mathrm{~mm}, y=1 \mathrm{~mm}$ ). From Fig. 3 and Fig.4, following observations are worth noting:

- The region surrounded by displacement-time curve of mode 1 is the largest, and frequencies of mode 2 and mode 3increase very significant, the regions surrounded by displacement-time curve are smaller.

- For different modes, the peak position and the vibration period are different, and the natural frequencies of higher modes are relatively large, the vibration periods decrease.

- The dynamic responses of non-linear dynamics appear asymmetric, and the displacement-time curve is no longer symmetrical about the system equilibrium position, this phenomenon is most obvious in the first mode. This is due to considering the non-linear factor of electrostatic force in the non-linear solution, and which causes the DC and harmonic component to the vibration response.

- For the micro plate simply supported on four sides, the boundary condition and material geometry are symmetry, the time response curve is symmetry too, the point of maximum displacement occurs at the center of the moving micro plate.

\section{CONCLUSIONS}

In this paper, non-linear dynamic responses of electromechanical coupled micro plate simply supported on four sides are analyzed. Control differential equation and vibration mode function for electromechanical coupled micro plate simply supported on four sides are presented. Defining the dynamic electrostatic force per unit area in Taylor series form, the forced non-linear dynamic equation is obtained. These works can provide theoretical basis for the parameters calculation of dynamic design and manufacture of MEMS typical components driving by electrostatic force.

The time dynamic responses are under the condition of no damp, which is the analysis result of ideal condition, and this work can provide a theoretical basis for the dynamic analysis of damping system.

\section{REFERENCES}

[1] Xu L, Sun L. Electromechanical coupled non-linear vibration of the micro plate. Proceedings of the Institution of Mechanical Engineers Part C-Journal of Mechanical Engineering Science, 2010, 224(C6): 13831396

[2] Libo Sun, Lizhong $\mathrm{Xu}$. Non-linear forced vibration for an electromechanical coupled micro plate simple supported at three sides and free at one side. 2009IEEE International Conference on Mechatronics and Automation, Changchun, 2009, 42(3): 4289-4293.

[3] Zafer Kazanc, Zahit Mecitoglu. Non-linear dynamic behavior of simply supported laminated composite plates subjected to blast load. Sound and Vibration, 2008,19(3):883 897

[4] Minhang Bao, Heng Yang. Squeeze film damping in MEMS. Sensors and Actuators, 2007,(136):3 27

[5] Liang YC, et al. A neural-network-based method of model reduction for the dynamic simulation of MEMS.J Micromechanics and Micro engineering, 2001, 11(3):226 233.

[6] Cleland A N, Roukes M L. Fabrication of high frequency nanometer scale mechanical resonators from bulk Si crystals. Appl Phys Lett, 1996,69(18):2653 2655

[7] Yang W.The mechanics characteristic of micro-nanoscale. World Scitech R \& D, 2004, 26(4):2 6.

[8] Wang chao,et al.Non-linear analysis of mechanical problems in MEMS application. Machine development, 2005, 34(8):18 20.

[9] Cao Zhiyuan.Vibration theory of plates and shells. Beijing: China's railway press, 1989:13 25.

[10] Herbert H W, James R M.Electromechanical dynamics. Beijing: China Machine Press, 1982:33 39.

[11] Liu Yanzhu.Mechanic of vibrations. Beijing:Higher Education Press, 1998:201 206. 